\title{
ON SOME PEXIDER-TYPE FUNCTIONAL EQUATIONS CONNECTED WITH THE ABSOLUTE VALUE OF ADDITIVE FUNCTIONS. PART II
}

\author{
BARBARA PRZEBIERACZ
}

(Received 2 March 2011)

\section{Abstract}

We investigate the Pexider-type functional equation

$$
\max \{f(x+y), f(x-y)\}=f(x) g(y)+h(y), \quad x, y \in G,
$$

where $f, g, h$ are real functions defined on an abelian group $G$. We solve this equation under the assumptions $G=\mathbb{R}$ and $f$ is continuous.

2010 Mathematics subject classification: primary 39B22; secondary 39 B52.

Keywords and phrases: Pexider functional equations, Cauchy equations, absolute value of additive functions.

\section{Introduction}

In [3] we introduced two Pexider functional equations:

$$
\max \{f(x+y), f(x-y)\}=f(x) g(y)+h(y)
$$

and

$$
\max \{f(x+y), f(x-y)\}=f(y) g(x)+h(x),
$$

with $f, g, h: G \rightarrow \mathbb{R}$, where $G$ is an abelian group. These are common generalizations of two functional equations:

$$
\max \{f(x+y), f(x-y)\}=f(x)+f(y)
$$

and

$$
\max \{f(x+y), f(x-y)\}=f(x) f(y) .
$$

For the solution of (1.2) see [3, Theorem 1.1]. Here, in Theorem 3.1, we are going to describe the solutions of (1.1) under the additional assumptions that $G=\mathbb{R}$ and $f$ is continuous. However, some results concerning this equation in more general settings, that is for an arbitrary abelian group $G$ and $f, g, h: G \rightarrow \mathbb{R}$, will be given in Section 2 .

This research was supported by the University of Silesia (Refinement Equations and Selected Topics in Iteration Theory Program).

(C) 2011 Australian Mathematical Publishing Association Inc. 0004-9727/2011 \$16.00 


\section{Equation (1.1) on groups}

As we will see, there is a relationship between solutions of (1.1) and (1.2). First, for a given $f: G \rightarrow \mathbb{R}$, we introduce the following functions:

$$
\begin{aligned}
F(x) & :=\max \{f(x), f(-x)\}, \quad x \in G ; \\
\tilde{f}(x) & :=f(x)-f(0), \quad x \in G ; \\
\tilde{F}(x) & :=\max \{\tilde{f}(x), \tilde{f}(-x)\}=F(x)-f(0), \quad x \in G .
\end{aligned}
$$

Of course, $F$ and $\tilde{F}$ are even; moreover, $\tilde{f}(0)=0$ and $\tilde{F}(0)=0$.

Lemma 2.1. Let $G$ be an abelian group, and let $f, g, h: G \rightarrow \mathbb{R}$ satisfy (1.1). Then

$$
\max \{\tilde{f}(x+y), \tilde{f}(x-y)\}=\tilde{f}(x) g(y)+\tilde{F}(y), \quad x, y \in G .
$$

Moreover, if $g \geq 0$ then $F, g, h$ and $\tilde{F}, g, \tilde{F}$ satisfy (1.1).

Proof. With $x=0$ in (1.1) we obtain

$$
\max \{f(y), f(-y)\}=f(0) g(y)+h(y),
$$

whence

$$
h(y)=F(y)-f(0) g(y), \quad y \in G .
$$

Therefore,

$$
\max \{f(x+y), f(x-y)\}=f(x) g(y)+F(y)-f(0) g(y),
$$

and by subtracting $f(0)$ from each side we get

$$
\max \{\tilde{f}(x+y), \tilde{f}(x-y)\}=\tilde{f}(x) g(y)+\tilde{F}(y) .
$$

Further, assume that $g \geq 0$. We have

$$
\begin{aligned}
\max \{F(x+y), F(x-y)\} & =\max \{\max \{f(x+y), f(-x-y)\}, \max \{f(x-y), f(-x+y)\}\} \\
& =\max \{\max \{f(x+y), f(x-y)\}, \max \{f(-x-y), f(-x+y)\}\} \\
& =\max \{f(x) g(y)+h(y), f(-x) g(y)+h(y)\} \\
& =\max \{f(x), f(-x)\} g(y)+h(y)=F(x) g(y)+h(y) .
\end{aligned}
$$

This implies

$$
\begin{aligned}
\max \{\tilde{F}(x+y), \tilde{F}(x-y)\} & =\max \{F(x+y), F(x-y)\}-f(0) \\
& =F(x) g(y)+h(y)-f(0)=F(x) g(y)+F(y)-f(0) g(y)-f(0) \\
& =\tilde{F}(x) g(y)+\tilde{F}(y) .
\end{aligned}
$$

This concludes the proof.

We proceed with the following simple observations.

Remark 2.2. Let $G$ be an abelian group and consider $f, g, h: G \rightarrow \mathbb{R}$. Then, $f, g, h$ satisfy (1.2) if and only if $f, g, h$ satisfy (1.1) and $f$ is even. 
Proof. First assume that $f, g, h$ satisfy (1.2). If $f$ is constant, $g$ is an arbitrary function and $h(x)=f(x)(1-g(x))$, then $f$ is obviously even and (1.1) is fulfilled by $f, g, h$. However, if $f$ is not constant, then $f, g, h$ are even (see [3, Remark 3.1]), and we have

$$
\begin{aligned}
\max \{f(x+y), f(x-y)\} & =\max \{f(-x-y), f(x-y)\} \\
& =f(x) g(-y)+h(-y)=f(x) g(y)+h(y) .
\end{aligned}
$$

Now, assume that $f, g, h$ satisfy (1.1) and $f$ is even. Then,

$$
\begin{aligned}
\max \{f(x+y), f(x-y)\} & =\max \{f(-x-y), f(x-y)\} \\
& =f(-y) g(x)+h(x)=f(y) g(x)+h(x) .
\end{aligned}
$$

This concludes the proof.

Remark 2.3. Let $G$ be an abelian group, and consider $x_{0} \in G$ and $f, g, h: G \rightarrow \mathbb{R}$. Then $f, g, h$ satisfy (1.1) if and only if $f\left(\cdot-x_{0}\right), g, h$ satisfy (1.1).

We can formulate the following corollary.

Corollary 2.4. Let $G$ be an abelian group, and consider $x_{0} \in G$ and $f, g, h: G \rightarrow \mathbb{R}$. If $f, g, h$ satisfy (1.2) then $f\left(\cdot-x_{0}\right), g, h$ satisfy (1.1).

In view of [3, Theorem 1.1] we have the following solutions of (1.1):

(1) $\left\{\begin{array}{l}f(x)=b, \\ g \text { is an arbitrary function, } \\ h(x)=b(1-g(x)),\end{array}\right.$

where $b \in \mathbb{R}$;

(2) $\left\{\begin{array}{l}f(x)=c \phi\left(x-x_{0}\right)+b, \\ g(x)=\phi(x), \\ h(x)=b(1-\phi(x)),\end{array}\right.$

where $x_{0}, c, b \in \mathbb{R}, c>0$, and $\phi: G \rightarrow \mathbb{R}$ is a solution of (1.4);

(3) $\left\{\begin{array}{l}f(x)=c \phi\left(x-x_{0}\right)+b, \\ g(x)=\phi(x), \\ h(x)=b(1-\phi(x)),\end{array}\right.$

where $x_{0}, c, b \in \mathbb{R}, c<0$, and $\phi: G \rightarrow \mathbb{R}$ is a solution of

$$
\min \{\phi(x+y), \phi(x-y)\}=\phi(x) \phi(y)
$$

(4) $\left\{\begin{array}{l}f(x)=\phi\left(x-x_{0}\right)+b, \\ g(x)=1, \\ h(x)=\phi(x),\end{array}\right.$

where $x_{0}, b \in \mathbb{R}$, and $\phi: G \rightarrow \mathbb{R}$ is a solution of (1.3). 
For solutions of (1.3), (1.4) and (2.3) see [3, Section 2]. Nevertheless, as we will see, these are not all solutions to (1.1).

Other partial results can be derived from [3, Theorem 1.1], Lemma 2.1 and Remark 2.2, as in the following corollary.

Corollary 2.5. Let $G$ be an abelian group and suppose that $f, g, h: G \rightarrow \mathbb{R}$ satisfy (1.1). Suppose also that $g \geq 0$. Then:

(1) $\left\{\begin{array}{l}F(x)=b \\ g \text { is an arbitrary function, } \\ h(x)=b(1-g(x)),\end{array}\right.$

where $b \in \mathbb{R}$; or

(2) $\left\{\begin{array}{l}F(x)=c \phi(x)+b, \\ g(x)=\phi(x), \\ h(x)=b(1-\phi(x)),\end{array}\right.$

where $c, b \in \mathbb{R}, c>0$, and $\phi: G \rightarrow \mathbb{R}$ is a solution of (1.4); or

(3) $\left\{\begin{array}{l}F(x)=c \phi(x)+b, \\ g(x)=\phi(x), \\ h(x)=b(1-\phi(x)),\end{array}\right.$

where $c, b \in \mathbb{R}, c<0$, and $\phi: G \rightarrow \mathbb{R}$ is a solution of (2.3); or

(4) $\left\{\begin{array}{l}F(x)=\phi(x)+b, \\ g(x)=1, \\ h(x)=\phi(x),\end{array}\right.$

where $b \in \mathbb{R}$, and $\phi: G \rightarrow \mathbb{R}$ is a solution of (1.3).

We finish this section with one more simple observation, in view of, for example, (2.1) and (2.2).

Corollary 2.6. Suppose that $f, g, h: G \rightarrow \mathbb{R}$ satisfy (1.1) and $f$ is not constant. Then $g(0)=1$ and $g$ and $h$ are even.

\section{Solution of (1.1) on $\mathbb{R}$}

In this section we are going to show the main result of this paper, that is, the following theorem.

Theorem 3.1. Let $f, g, h: \mathbb{R} \rightarrow \mathbb{R}$ satisfy equation (1.1) and assume that $f$ is continuous. Then the functions $f, g$, $h$ are of one of the following forms:

(1) $\left\{\begin{array}{l}f(x)=b \\ g \text { is an arbitrary function, } \\ h(x)=b(1-g(x))\end{array}\right.$

where $b \in \mathbb{R}$; 
(2) $\left\{\begin{array}{l}f(x)=c e^{a\left|x-x_{0}\right|}+b, \\ g(x)=e^{a|x|} \\ h(x)=b\left(1-e^{a|x|}\right)\end{array}\right.$

where $x_{0}, b \in \mathbb{R}, a c>0$;

(3) $\left\{\begin{array}{l}f(x)=a\left|x-x_{0}\right|+b \\ g(x)=1 \\ h(x)=a|x|\end{array}\right.$

where $b, x_{0} \in \mathbb{R}, a>0$;

(4) $\left\{\begin{array}{l}f(x)=c e^{a x}+b \\ g(x)=e^{\operatorname{sgn}(c)|a x|} \\ h(x)=b\left(1-e^{\operatorname{sgn}(c)|a x|}\right)\end{array}\right.$

where $a, b, c \in \mathbb{R}$;

(5) $\left\{\begin{array}{l}f(x)=a x+b \\ g(x)=1 \\ h(x)=|a x|\end{array}\right.$

where $a, b \in \mathbb{R}$.

Conversely, if $f, g$, $h$ are of one of the forms (1)-(5), then they satisfy (1.1).

The 'if' part of this theorem (the converse) can be checked directly. The remainder of the paper is concerned with proving that if $f, g, h: \mathbb{R} \rightarrow \mathbb{R}$ satisfy (1.1) and $f$ is continuous then $f, g, h$ are of the forms (1)-(5). First notice that if $f \equiv b$ is constant and $g$ is an arbitrary function then $h(y):=b(1-g(y))$. So, from now on, we will work under the assumptions

$$
f, g, h: \mathbb{R} \rightarrow \mathbb{R} \text {, satisfy (1.1), and } f \text { is continuous and not constant. }
$$

Remark 3.2. Suppose (H). Then the functions $g$ and $h$ are continuous.

Proof. By (2.1) with $x_{0} \in \mathbb{R}$ such that $\tilde{f}\left(x_{0}\right) \neq 0$,

$$
g(y)=\frac{\max \left\{\tilde{f}\left(x_{0}+y\right), \tilde{f}\left(x_{0}-y\right)\right\}-\tilde{F}(0)}{\tilde{f}\left(x_{0}\right)} .
$$

Therefore $g$ is continuous. Moreover, from (2.2), $h$ is continuous, too.

Lemma 3.3. Suppose (H). Then $g \geq 0$.

Proof. We consider three cases.

(I) $\tilde{F} \equiv 0$. Since $f$ is not constant, we can find an $x_{0} \neq 0$ such that $\tilde{f}\left(x_{0}\right)<0$. The inequality $g(y)<0$ for some $y \in \mathbb{R}$ implies, by (2.1),

$$
0 \geq \max \left\{\tilde{f}\left(x_{0}+y\right), \tilde{f}\left(x_{0}-y\right)\right\}=\tilde{f}\left(x_{0}\right) g(y)+\tilde{F}(y)=\tilde{f}\left(x_{0}\right) g(y)>0,
$$

which is impossible. Hence, $g \geq 0$. 
(II) $\tilde{F} \leq 0$ and $\tilde{F} \not \equiv 0$. Suppose that $g(y)=0$ for some $y \in \mathbb{R}$. Since $g(0)=1$ and $g$ is continuous and even, we can define $y_{0}:=\min \{y>0: g(y)=0\}$. We have, using (2.1) again,

$$
\max \left\{\tilde{f}\left(x+y_{0}\right), \tilde{f}\left(x-y_{0}\right)\right\}=\tilde{f}(x) g\left(y_{0}\right)+\tilde{F}\left(y_{0}\right)=\tilde{F}\left(y_{0}\right), \quad x \in \mathbb{R} .
$$

Putting $x=-y_{0}$ in the above, we obtain

$$
0=\tilde{f}(0) \leq \max \left\{\tilde{f}(0), \tilde{f}\left(x-y_{0}\right)\right\}=\tilde{F}\left(y_{0}\right) \leq 0 .
$$

Hence we have proved that

$$
\tilde{F}\left(y_{0}\right)=0 .
$$

Now we will prove the implication

$$
\tilde{F}(x)<0 \Rightarrow \forall_{z \in\left(x-y_{0}, x+y_{0}\right)} \tilde{f}(z)<0 .
$$

Fix an $x$ with $\tilde{F}(x)<0$ and $y \in\left(-y_{0}, y_{0}\right)$. By (2.1) we have

$$
\max \{\tilde{f}(x+y), \tilde{f}(x-y)\}=\tilde{f}(x) g(y)+\tilde{F}(y)<0,
$$

as $\tilde{f}(x)<0, g(y)>0$ and $\tilde{F}(y) \leq 0$. Therefore, we have proved (3.3).

Now fix an $x_{0}$ such that $\tilde{F}\left(x_{0}\right)<0$. By (3.1) and (3.2) we have

$$
\max \left\{\tilde{f}\left(x_{0}+y_{0}\right), \tilde{f}\left(x_{0}-y_{0}\right)\right\}=0 .
$$

Suppose, without loss of generality, that

$$
\tilde{f}\left(x_{0}-y_{0}\right)=0 .
$$

For an arbitrary $x_{1} \in\left(x_{0}-y_{0}, x_{0}\right)$ we have $\tilde{f}\left(x_{1}\right)<0$ (see (3.3)). Hence, using (3.3) for $x_{1}$ we infer that $\tilde{f}(z)<0$ for $z \in\left(x_{1}-y_{0}, x_{1}+y_{0}\right)$. But $x_{0}-y_{0} \in\left(x_{1}-y_{0}, x_{1}+y_{0}\right)$, so $\tilde{f}\left(x_{0}-y_{0}\right)<0$, which is a contradiction with (3.4). Therefore $g>0$.

(III) $\tilde{F}\left(z_{0}\right)>0$ for some $z_{0} \in \mathbb{R}$. If $g>0$ then the proof is finished. So, assume that it is not the case. Then, by continuity of $g$, the fact that $g$ is even and $g(0)=1$, we can define

$$
y_{0}:=\min \{y>0: g(y)=0\} .
$$

Put $M:=\sup \{\tilde{f}(x): x \in \mathbb{R}\}$. We have, of course, $M>0$. Moreover,

$$
M=\tilde{F}(y), \quad y \in g^{-1}(\{0\}) .
$$

Indeed, by (2.1),

$$
\max \{\tilde{f}(x+y), \tilde{f}(x-y)\}=\tilde{f}(x) g(y)+\tilde{F}(y)=\tilde{F}(y), \quad x \in \mathbb{R}, y \in g^{-1}(\{0\}) .
$$

This implies

$$
\tilde{f}(x) \leq \tilde{F}(y), \quad x \in \mathbb{R}, y \in g^{-1}(\{0\}) .
$$

Hence $M \leq \tilde{F}(y)$, for every $y \in g^{-1}(\{0\})$. Therefore, by the definition of $M$ and $\tilde{F}$ we infer that $M=\tilde{F}(y)$, for every $y \in g^{-1}(\{0\})$. Now, (3.6) implies

$$
\left[\tilde{f}(x)<M \text { and } y \in g^{-1}(\{0\})\right] \Rightarrow \tilde{f}(x+2 y)=\tilde{f}(x-2 y)=M .
$$


In particular, since $\tilde{f}(0)=0<M$,

$$
\tilde{f}(2 y)=\tilde{f}(-2 y)=M, \quad y \in g^{-1}(\{0\}) .
$$

Put

$$
\begin{aligned}
& \delta:=\min \{\varepsilon>0: \tilde{f}(-\varepsilon)=M\}, \\
& \eta:=\min \{\varepsilon>0: \tilde{f}(\varepsilon)=M\} .
\end{aligned}
$$

This means that $\tilde{f}(-\delta)=\tilde{f}(\eta)=M$ and $\tilde{f}(x)<M$ for $x \in(-\delta, \eta)$. We also have

$$
\tilde{F}(-\delta)=\tilde{F}(\eta)=M
$$

By (3.7), $\tilde{f}\left(x+2 y_{0}\right)=M$ for every $x \in(-\delta, \eta)$. Hence $\tilde{F}\left(x+2 y_{0}\right)=M$, for $x \in(-\delta, \eta)$. Continuity of $\tilde{F}$ assures us that we also have

$$
\tilde{F}\left(2 y_{0}-\delta\right)=\tilde{F}\left(2 y_{0}+\eta\right)=M
$$

Using (2.1) we get

$$
\max \left\{\tilde{f}\left(2 y_{0}+\eta\right), \tilde{f}\left(2 y_{0}-\eta\right)\right\}=\tilde{f}\left(2 y_{0}\right) g(\eta)+\tilde{F}(\eta) .
$$

This, together with (3.10), (3.8) and (3.9), gives $g(\eta)=0$. However, from the definition of $y_{0}$, this means that $y_{0} \leq \eta$, but, from the definition of $\eta$,

$$
\tilde{f}(x)<M, \quad x \in\left[0, y_{0}\right) .
$$

Similarly,

$$
\max \left\{\tilde{f}\left(2 y_{0}+\delta\right), \tilde{f}\left(2 y_{0}-\delta\right)\right\}=\tilde{f}\left(2 y_{0}\right) g(-\delta)+\tilde{F}(-\delta) .
$$

This, together with (3.10), (3.8) and (3.9), gives $g(-\delta)=0$. However, from the definition of $y_{0}$, this means that $y_{0}<\delta$, but, from the definition of $\delta$,

$$
\tilde{f}(x)<M, \quad x \in\left(-y_{0}, 0\right) .
$$

This, together with (3.11), means that $\tilde{f}(x)<M$ for every $x \in\left(-y_{0}, y_{0}\right)$. Now, in view of (3.7) (and the continuity of $\tilde{f}$ ), we infer

$$
\tilde{f}(x)=M, \quad x \in\left[y_{0}, 3 y_{0}\right] .
$$

On account of (2.1),

$$
\max \left\{\tilde{f}\left(3 y_{0}-x\right), \tilde{f}\left(3 y_{0}+x\right)\right\}=\tilde{f}\left(3 y_{0}\right) g(x)+\tilde{F}(x), \quad x \in \mathbb{R},
$$

and hence, by (3.12),

$$
g(x)=0, \quad x \in\left[y_{0}, 2 y_{0}\right] .
$$

Moreover, from the definition of $y_{0}$ and $g(0)=1$, we know that

$$
g(x)>0, \quad x \in\left[0, y_{0}\right) .
$$

Now, we will prove the implication

$$
g(y)=0 \Rightarrow g(2 y)=0, \quad y \in \mathbb{R} .
$$


Notice that in view of (3.13), (3.15) together with (3.14) (and the fact that $g$ is even), implies that $g(x)>0$ for $x \in\left(-y_{0}, y_{0}\right)$ and $g(x)=0$ for $x \in\left(-\infty,-y_{0}\right] \cup\left[y_{0}, \infty\right)$. Hence, proving (3.15) will complete the proof.

Suppose that $g(y)=0$. By (3.5) we have $\tilde{F}(y)=M$. Let $x \in \mathbb{R}$ be such that $0<\tilde{f}(x)<M$ (such an $x$ exists, since $\tilde{f}(0)=0$ and $\tilde{F}\left(y_{0}\right)=M$ ). Therefore, using (2.1),

$$
\max \{\tilde{f}(x), \tilde{f}(x+2 y)\}=\tilde{f}(x+y) g(y)+\tilde{F}(y) .
$$

This implies $\tilde{f}(x+2 y)=M$, since $\tilde{f}(x)<M, g(y)=0$ and $\tilde{F}(y)=M$. Using (2.1) again, we obtain

$$
\max \{\tilde{f}(x+2 y), \tilde{f}(x-2 y)\}=\tilde{f}(x) g(2 y)+\tilde{F}(2 y) .
$$

Consequently, $g(2 y)=0$, since $\tilde{f}(x+2 y)=M, \tilde{f}(x)>0$ and $\tilde{F}(2 y)=M$ (see (3.8)). The proof of (3.15) is complete.

\section{Lemma 3.4. Suppose (H). Then $\tilde{F}$ is not constant.}

Proof. Suppose, on the contrary, that $\tilde{F} \equiv 0$. Since $f$ is not constant, there exists an $x_{0} \neq 0$ such that $\tilde{f}\left(x_{0}\right)<0$. Without loss of generality, we can assume that $x_{0}>0$. Let $z_{0} \geq 0$ be such that $\tilde{f}\left(z_{0}\right)=0$ and $\tilde{f}(x)<0$ for every $x \in\left(z_{0}, x_{0}\right]$. For an arbitrary $x \in\left(z_{0}, x_{0}\right]$, by $(2.1)$,

$$
0=\max \left\{\tilde{f}\left(2 x-z_{0}\right), \tilde{f}\left(z_{0}\right)\right\}=\tilde{f}(x) g\left(x-z_{0}\right)+\tilde{F}\left(x-z_{0}\right)=\tilde{f}(x) g\left(x-z_{0}\right) .
$$

In view of $\tilde{f}(x)<0$ we infer $g\left(x-z_{0}\right)=0$. Passing with $x$ to $z_{0}$, on account of the continuity of $g$, gives $g(0)=0$. However, we know from Corollary 2.6 that $g(0)=1$. This is a contradiction.

We now reformulate Corollary 2.5 under the assumption (H) (see [3, Section 2]).

Corollary 3.5. Suppose (H). Then we have one of the following cases:

$$
\left\{\begin{array}{l}
\tilde{F}(x)=C\left(e^{A|x|}-1\right) \\
F(x)=C\left(e^{A|x|}-1\right)+f(0) \\
g(x)=e^{A|x|} \\
h(x)=(f(0)-C)\left(1-e^{A|x|}\right)
\end{array}\right.
$$

where $A, C>0$;

(V) $\left\{\begin{array}{l}\tilde{F}(x)=A|x| \\ F(x)=A|x|+f(0), \\ g(x)=1 \\ h(x)=A|x|\end{array}\right.$

where $A>0$;

$(\mathrm{W})\left\{\begin{array}{l}\tilde{F}(x)=C\left(1-e^{-A|x|}\right), \\ F(x)=C\left(1-e^{-A|x|}\right)+f(0), \\ g(x)=e^{-A|x|} \\ h(x)=(f(0)+C)\left(1-e^{-A|x|}\right),\end{array}\right.$

where $A, C>0$. 
In particular, $g(x)>0, x \in \mathbb{R}$, and $F$ and $\tilde{F}$ are strictly decreasing on $(-\infty, 0]$ and strictly increasing on $[0, \infty)$.

We see that it is enough to determine the formula for $f$ in each of the cases (U), (V) and (W). Now, we are going to prove four technical lemmas, which will be summarized in Corollary 3.10.

Lemma 3.6. Suppose (H). Then we have the following results.

- If there exists an $x_{0}<0$ such that $\tilde{f}\left(x_{0}\right)<\tilde{F}\left(x_{0}\right)$, then

$$
\begin{aligned}
& \tilde{f}(x)<\tilde{F}(x), \quad x \in(-\infty, 0), \\
& \tilde{f}(x)=\tilde{F}(x), \quad x \in[0, \infty) .
\end{aligned}
$$

- If there exists an $x_{0}>0$ such that $\tilde{f}\left(x_{0}\right)<\tilde{F}\left(x_{0}\right)$, then

$$
\begin{array}{ll}
\tilde{f}(x)<\tilde{F}(x), & x \in(0, \infty), \\
\tilde{f}(x)=\tilde{F}(x), & x \in(-\infty, 0] .
\end{array}
$$

Proof. Let us assume that there exists an $x_{0}<0$ such that $\tilde{f}\left(x_{0}\right)<\tilde{F}\left(x_{0}\right)$ (the second implication can be considered analogously). Suppose that there is a $y<x_{0}$ such that $\tilde{f}(y)=\tilde{F}(y)$. We can choose $x_{1}<0$ and $y_{1}>0$ with $x_{1}+y_{1}<0, \tilde{f}\left(x_{1}\right)<\tilde{F}\left(x_{1}\right)$ and $\tilde{f}\left(x_{1}-y_{1}\right)=\tilde{F}\left(x_{1}-y_{1}\right)$. Using (2.1), the positiveness of $g$, the fact that $\tilde{F}, g, \tilde{F}$ satisfy (1.1) and the monotonicity of $\tilde{F}$, we have

$$
\begin{aligned}
\tilde{F}\left(x_{1}-y_{1}\right) & =\tilde{f}\left(x_{1}-y_{1}\right) \leq \max \left\{\tilde{f}\left(x_{1}+y_{1}\right), \tilde{f}\left(x_{1}-y_{1}\right)\right\} \\
& =\tilde{f}\left(x_{1}\right) g\left(y_{1}\right)+\tilde{F}\left(y_{1}\right)<\tilde{F}\left(x_{1}\right) g\left(y_{1}\right)+\tilde{F}\left(y_{1}\right) \\
& =\max \left\{\tilde{F}\left(x_{1}+y_{1}\right), \tilde{F}\left(x_{1}-y_{1}\right)\right\}=\tilde{F}\left(x_{1}-y_{1}\right) .
\end{aligned}
$$

This is a contradiction. Hence, we have proved that if $\tilde{f}(x)<\tilde{F}(x)$, for some $x<0$, then $\tilde{f}(y)<\tilde{F}(y)$, for every $y<x$. Let $z:=\sup \{x<0: \tilde{f}(x)<\tilde{F}(x)\}$. We have

$$
\tilde{f}(z)=\tilde{F}(z)
$$

and

$$
\tilde{f}(x)<\tilde{F}(x), \quad x<z .
$$

Let $y>-2 z$. Hence, $-z-y<z \leq 0$. Therefore, $\tilde{f}(-z-y)<\tilde{F}(-z-y)=\tilde{F}(z+y)$. Hence,

$$
\tilde{f}(z+y)=\tilde{F}(z+y) .
$$

Using the inequalities $0<z+y \leq y-z$, the fact that $\tilde{F}$ is even and the results concerning its monotonicity from Corollary 3.5 , we obtain

$$
\tilde{F}(z+y) \leq \tilde{F}(z-y)
$$

Suppose that

$$
\tilde{F}(z+y)<\tilde{F}(z-y)
$$


Using (2.1), (3.16), Lemma 2.1 and (3.19), we obtain

$$
\begin{aligned}
\max \{\tilde{f}(z+y), \tilde{f}(z-y)\} & =\tilde{f}(z) g(y)+\tilde{F}(y) \\
& =\tilde{F}(z) g(y)+\tilde{F}(y)=\max \{\tilde{F}(z+y), \tilde{F}(z-y)\}=\tilde{F}(z-y) .
\end{aligned}
$$

However, since we have shown (3.18) and assumed (3.20), then it follows that $\tilde{f}(z-y)=\tilde{F}(z-y)$, which is impossible, as $z-y<z$ and (3.17) holds. Therefore, (3.20) leads to a contradiction. Hence, in view of this and (3.19) we find $\tilde{F}(z+y)=\tilde{F}(z-y)$, which, due to the properties of $\tilde{F}$, implies $z=0$. Hence, by (3.17), we have proved that $\tilde{f}(x)<\tilde{F}(x)$, for $x<0$ and, consequently, $\tilde{f}(x)=\tilde{F}(x)$, for $x \geq 0$.

Lemma 3.7. Suppose (H). For any $c \in \mathbb{R}$ define function $H_{c}: \mathbb{R} \rightarrow \mathbb{R}$ by the formula $H_{c}(x):=\operatorname{cg}(x)+\tilde{F}(x)$. Then we have the following results.

- $\quad$ For every $x_{0}<0$ and $c \in\left[0, \tilde{F}\left(x_{0}\right)\right]$,

$$
H_{c}\left(x-x_{0}\right)>\tilde{F}(x), \quad x \geq 0 .
$$

- $\quad$ For every $x_{0}>0$ and $c \in\left[0, \tilde{F}\left(x_{0}\right)\right]$,

$$
H_{c}\left(x-x_{0}\right)>\tilde{F}(x), \quad x \leq 0 .
$$

Proof. Using the formulas for $g$ and $\tilde{F}$ from Corollary 3.5 , we can directly check the inequalities in each of the cases (U), (V) and (W). We omit the calculations here.

Lemma 3.8. Suppose (H). Then the following implications hold true.

- If there exist $x_{0}, x_{1}<0$ such that $\tilde{f}\left(x_{0}\right) \geq 0$ and $\tilde{f}\left(x_{1}\right)<\tilde{F}\left(x_{1}\right)$, then $\tilde{f}\left(x_{0}-y\right)=$ $H_{\tilde{f}\left(x_{0}\right)}(y)$ for $y \geq\left|x_{0}\right|$.

- If there exist $x_{0}, x_{1}>0$ such that $\tilde{f}\left(x_{0}\right) \geq 0$ and $\tilde{f}\left(x_{1}\right)<\tilde{F}\left(x_{1}\right)$, then $\tilde{f}\left(x_{0}+y\right)=$ $H_{\tilde{f}\left(x_{0}\right)}(y)$ for $y \geq\left|x_{0}\right|$.

PROOF. We will prove only the first part. From Lemma 3.6 we infer

$$
\tilde{f}(x)=\tilde{F}(x), \quad x \in[0, \infty) .
$$

Fix $y \geq\left|x_{0}\right|$. Using (2.1) we obtain

$$
\max \left\{\tilde{f}\left(x_{0}-y\right), \tilde{f}\left(x_{0}+y\right)\right\}=\tilde{f}\left(x_{0}\right) g(y)+\tilde{F}(y)=H_{\tilde{f}\left(x_{0}\right)}(y) .
$$

Since $x_{0}+y \geq 0$, by (3.21) and Lemma 3.7 we obtain

$$
\tilde{f}\left(x_{0}+y\right)=\tilde{F}\left(x_{0}+y\right)<H_{\tilde{f}\left(x_{0}\right)}\left(x_{0}+y-x_{0}\right)=H_{\tilde{f}\left(x_{0}\right)}(y),
$$

which together with (3.22) gives

$$
\tilde{f}\left(x_{0}-y\right)=H_{\tilde{f}\left(x_{0}\right)}(y) .
$$

This concludes the proof. 
Lemma 3.9. Suppose (H). The following implications hold true.

- If there exists a $y<0$ such that $\tilde{f}(y)<\tilde{F}(y)$ then $\tilde{f}(x)<0$ for $x \in(-\delta, 0)$ for some $\delta>0$.

- If there exists a $y>0$ such that $\tilde{f}(y)<\tilde{F}(y)$ then $\tilde{f}(x)<0$ for $x \in(0, \delta)$ for some $\delta>0$.

Proof. Again, we will prove the first part only. From Lemma 3.6 we infer

$$
\tilde{f}(x)<\tilde{F}(x), \quad x \in(-\infty, 0) .
$$

Suppose, on the contrary, that there is an increasing sequence $\left(x_{n}: n \in \mathbb{N}\right)$ tending to 0 as $n \rightarrow \infty$, and such that $\tilde{f}\left(x_{n}\right) \geq 0$ for $n \in \mathbb{N}$. Choose $z \leq 2 x_{1}$. We have $x_{n}-z \geq\left|x_{n}\right|$, $n \in \mathbb{N}$. Using Lemma 3.8 we obtain

$$
\tilde{f}(z)=H_{\tilde{f}\left(x_{n}\right)}\left(x_{n}-z\right)=\tilde{f}\left(x_{n}\right) g\left(x_{n}-z\right)+\tilde{F}\left(x_{n}-z\right) .
$$

Letting $n \rightarrow \infty$ in the above, we get $\tilde{f}(z)=\tilde{F}(z)$, contrary to (3.23).

Up to now, we have shown the following corollary.

Corollary 3.10. Suppose (H). Then we have the following possibilities:

$$
\begin{gathered}
\tilde{f}=\tilde{F} ; \\
\begin{cases}\tilde{f}(x)=\tilde{F}(x), & x \in[0, \infty), \\
\tilde{f}(x)<\tilde{F}(x), & x \in(-\infty, 0), \\
\tilde{f}(x)<0, & x \in(-\delta, 0), \text { for some } \delta>0 ;\end{cases} \\
\begin{cases}\tilde{f}(x)=\tilde{F}(x), & x \in(-\infty, 0], \\
\tilde{f}(x)<\tilde{F}(x), & x \in(0, \infty), \\
\tilde{f}(x)<0, & x \in(0, \delta), \text { for some } \delta>0 .\end{cases}
\end{gathered}
$$

In view of Corollary 3.5 we can consider further only cases (ii) and (iii). Moreover, in each case we distinguish two possibilities:

(1) either $\tilde{f}$ is negative on the whole half line $\mathbb{R}_{-}, \mathbb{R}_{+}$, respectively; or

(2) it is not.

Finally, we will determine the formula for $\tilde{f}$ (and $f$ ) according to cases (U), (V) and (W). Namely, we consider the following possibilities.

(1U(ii)) $\tilde{F}, F, g$ and $h$ are given by (U), (ii) holds and $\tilde{f}(x)<0$ for every $x \in(-\infty, 0)$.

(1V(ii)) $\tilde{F}, F, g$ and $h$ are given by (V), (ii) holds and $\tilde{f}(x)<0$ for every $x \in(-\infty, 0)$.

(1W(ii)) $\tilde{F}, F, g$ and $h$ are given by (W), (ii) holds and $\tilde{f}(x)<0$ for every $x \in(-\infty, 0)$.

(1U(iii)) $\tilde{F}, F, g$ and $h$ are given by (U), (iii) holds and $\tilde{f}(x)<0$ for every $x \in(0, \infty)$.

(1V(iii)) $\tilde{F}, F, g$ and $h$ are given by (V), (iii) holds and $\tilde{f}(x)<0$ for every $x \in(0, \infty)$.

(1W(iii)) $\tilde{F}, F, g$ and $h$ are given by (W), (iii) holds and $\tilde{f}(x)<0$ for every $x \in(0, \infty)$.

(2U(ii)) $\tilde{F}, F, g$ and $h$ are given by (U), (ii) holds and there is $x<0$ such that $\tilde{f}(x)=0$. 
(2V(ii)) $\tilde{F}, F, g$ and $h$ are given by (V), (ii) holds and there is $x<0$ such that $\tilde{f}(x)=0$.

(2W(ii)) $\tilde{F}, F, g$ and $h$ are given by (W), (ii) holds and there is $x<0$ such that $\tilde{f}(x)=0$.

(2U(iii)) $\tilde{F}, F, g$ and $h$ are given by (U), (iii) holds and there is $x>0$ such that $\tilde{f}(x)=0$.

(2V(iii)) $\tilde{F}, F, g$ and $h$ are given by (V), (iii) holds and there is $x>0$ such that $\tilde{f}(x)=0$.

(2W(iii)) $\tilde{F}, F, g$ and $h$ are given by (W), (iii) holds and there is $x>0$ such that $\tilde{f}(x)=0$.

First, we deal with (1U(ii)). Fix $x<0$. By (2.1) we obtain

$$
\max \{\tilde{f}(2 x), \tilde{f}(0)\}=\tilde{f}(x) g(x)+\tilde{F}(x),
$$

whence, since $\tilde{f}(2 x)<0=\tilde{f}(0)$ and $g(x)>0$,

$$
\tilde{f}(x)=-\frac{\tilde{F}(x)}{g(x)}, \quad x<0 .
$$

Therefore,

$$
\tilde{f}(x)=-\frac{C e^{A|x|}-C}{e^{A|x|}}=-C+C e^{A x}, \quad x<0
$$

but

$$
\tilde{f}(x)=\tilde{F}(x), \quad x \geq 0,
$$

so

$$
\begin{gathered}
\tilde{f}(x)=-C+C e^{A x}, \quad x \in \mathbb{R}, \\
f(x)=-C+C e^{A x}+f(0), \quad x \in \mathbb{R} .
\end{gathered}
$$

Similarly, using formulas (3.24) and (3.25), we obtain

$$
f(x)=A x+f(0), \quad x \in \mathbb{R},
$$

(1W(ii))

$$
f(x)=C-C e^{-A x}+f(0), \quad x \in \mathbb{R} .
$$

Analogously, one can show that in cases (1U(iii)), (1V(iii)) and (1W(iii))

$$
\tilde{f}(x)=-\frac{\tilde{F}(x)}{g(x)}, \quad x>0,
$$

and

$$
\tilde{f}(x)=\tilde{F}(x), \quad x \leq 0
$$

hence

(1U(iii))

$$
f(x)=-C+C e^{-A x}+f(0), \quad x \in \mathbb{R},
$$


(1V(iii))

$$
f(x)=-A x+f(0), \quad x \in \mathbb{R},
$$

(1W(iii))

$$
f(x)=C-C e^{A x}+f(0), \quad x \in \mathbb{R} .
$$

Compare points (4) and (5) from Theorem 3.1.

Secondly, we will consider case (2U(ii)). Put

$$
x_{0}:=\frac{1}{2} \max \{x<0: \tilde{f}(x)=0\} .
$$

Of course, $x_{0}<0, \tilde{f}\left(2 x_{0}\right)=0$ and $\tilde{f}(x)<0$ for $x \in\left(2 x_{0}, 0\right)$. We will calculate the formula for $\tilde{f}$ in a few steps.

- We already know that

$$
\tilde{f}(x)=\tilde{F}(x), \quad x \geq 0 .
$$

- $\quad$ Suppose $x<2 x_{0}$. Notice that

$$
\tilde{f}\left(2 x_{0}+y\right)<\tilde{F}(y), \quad y>0 .
$$

Indeed, either $y \in\left(0,-2 x_{0}\right)$ and then $2 x_{0}+y<0$, whence

$$
\tilde{f}\left(2 x_{0}+y\right)<0<\tilde{F}(y)
$$

or $y \geq-2 x_{0}$ and then $2 x_{0}+y \geq 0$, so consequently

$$
\tilde{f}\left(2 x_{0}+y\right)=\tilde{F}\left(2 x_{0}+y\right)<\tilde{F}(y),
$$

as $\tilde{F}$ increases on $[0, \infty)$.

From (2.1) we have

$$
\max \left\{\tilde{f}\left(2 x_{0}+y\right), \tilde{f}\left(2 x_{0}-y\right)\right\}=\tilde{f}\left(2 x_{0}\right) g(y)+\tilde{F}(y)=\tilde{F}(y) .
$$

On account of (3.27) we infer

$$
\tilde{f}\left(2 x_{0}-y\right)=\tilde{F}(y), \quad y>0,
$$

which can be reformulated as

$$
\tilde{f}(x)=\tilde{F}\left(2 x_{0}-x\right), \quad x<2 x_{0} .
$$

Therefore,

$$
\tilde{f}(x)=C\left(e^{A\left|2 x_{0}-x\right|}-1\right), \quad x<2 x_{0} .
$$

- $\quad$ Suppose $x \in\left(x_{0}, 0\right)$. Choose $y \in\left(-x, x-2 x_{0}\right)$. By (2.1) we have

$$
\max \{\tilde{f}(x+y), \tilde{f}(x-y)\}=\tilde{f}(x) g(y)+\tilde{F}(y) .
$$

Since $x+y>0$ we have $\tilde{f}(x+y)=\tilde{F}(x+y)>0$; moreover, $x-y \in\left(2 x_{0}, 0\right)$, and hence $\tilde{f}(x-y)<0$. Therefore, (3.29) implies

$$
\tilde{F}(x+y)=\tilde{f}(x) g(y)+\tilde{F}(y) .
$$

Consequently,

$$
\tilde{f}(x)=\frac{\tilde{F}(x+y)-\tilde{F}(y)}{g(y)}, \quad x \in\left(x_{0}, 0\right), y \in\left(-x, x-2 x_{0}\right) .
$$


Hence

$$
\tilde{f}(x)=C\left(e^{A x}-1\right), \quad x \in\left(x_{0}, 0\right) .
$$

- $\quad$ Suppose $x \in\left(2 x_{0}, x_{0}\right)$ and choose $y \in\left(x-2 x_{0},-x\right)$. Then

$$
\max \{\tilde{f}(x+y), \tilde{f}(x-y)\}=\tilde{f}(x) g(y)+\tilde{F}(y) .
$$

Hence, because $\tilde{f}(x+y)<0$ (since $x+y \in\left(2 x_{0}, 0\right)$ ) and $\tilde{f}(x-y)>0$ (we have $x-y$ $<2 x_{0}$, and so $\left.\tilde{f}(x-y)=\tilde{F}\left(2 x_{0}-(x-y)\right)>0\right)$, we infer $\tilde{f}(x-y)=\tilde{f}(x) g(y)+\tilde{F}(y)$. Therefore,

$$
\tilde{f}(x)=\frac{\tilde{F}\left(2 x_{0}-(x-y)\right)-\tilde{F}(y)}{g(y)}, \quad x \in\left(2 x_{0}, x_{0}\right), y \in\left(x-2 x_{0},-x\right) .
$$

We can calculate that

$$
\tilde{f}(x)=C\left(e^{A\left(x_{0}-x\right)}-1\right), \quad x \in\left(2 x_{0}, x_{0}\right) .
$$

- $\quad$ Of course, we also have $\tilde{f}\left(x_{0}\right)=C\left(e^{A x_{0}}-1\right)$.

Finally, we can summarize the formula for $\tilde{f}$ :

$$
\tilde{f}(x)=C\left(e^{A\left(\left|x-x_{0}\right|+x_{0}\right)}-1\right), \quad x \in \mathbb{R} .
$$

Therefore,

$$
f(x)=C\left(e^{A\left(\left|x-x_{0}\right|+x_{0}\right)}-1\right)+f(0), \quad x \in \mathbb{R} .
$$

Similarly, in cases (2V(ii)) and (2W(iii)), using (3.26), (3.28), (3.30) and (3.31), we obtain

$$
f(x)=A\left(\left|x-x_{0}\right|+x_{0}\right)+f(0), \quad x \in \mathbb{R},
$$

(2W(ii))

$$
f(x)=C\left(1-e^{-A\left(\left|x-x_{0}\right|+x_{0}\right)}\right)+f(0), \quad x \in \mathbb{R} .
$$

Analogously, in cases (2U(iii)), (2V(iii)) and $(2 \mathrm{~W}($ iii $))$, with $x_{0}:=\frac{1}{2} \min \{x>0$ : $\tilde{f}(x)=0\}$, we get the formulas:

$$
\begin{gathered}
\tilde{f}(x)=\tilde{F}(x), \quad x \leq 0 ; \\
\tilde{f}(x)=\tilde{F}\left(x-2 x_{0}\right), \quad x>2 x_{0} ; \\
\tilde{f}(x)=\frac{\tilde{F}(x-y)-\tilde{F}(y)}{g(y)}, \quad x \in\left(0, x_{0}\right), y \in\left(x, 2 x_{0}-x\right) ; \\
\tilde{f}(x)=\frac{\tilde{F}\left(x+y-2 x_{0}\right)-\tilde{F}(y)}{g(y)}, \quad x \in\left(x_{0}, 2 x_{0}\right), y \in\left(2 x_{0}-x, x\right) .
\end{gathered}
$$

Hence,

(2U(iii))

$$
f(x)=C\left(e^{A\left(\left|x-x_{0}\right|-x_{0}\right)}-1\right)+f(0), \quad x \in \mathbb{R},
$$


(2V(iii))

$$
f(x)=A\left(\left|x-x_{0}\right|-x_{0}\right)+f(0), \quad x \in \mathbb{R},
$$

(2W(iii))

$$
f(x)=C\left(1-e^{-A\left(\left|x-x_{0}\right|-x_{0}\right)}\right)+f(0), \quad x \in \mathbb{R} .
$$

Compare points (2) and (3) from Theorem 3.1.

This completes the proof.

\section{References}

[1] J. Aczél and J. Dhombres, Functional Equations in Several Variables, Encyclopedia of Mathematics and its Applications, 31 (Cambridge University Press, Cambridge, 1989).

[2] K. Baron and P. Volkmann, 'Characterization of the absolute value of complex linear functionals by functional equations', Seminar LV, No. 28 (2006), 10 pp., http://www.math.us.edu.pl/smdk.

[3] B. Przebieracz, 'On some Pexider-type functional equations connected with the absolute value of additive functions, Part I', Bull. Aust. Math. Soc. 85(2) (2012), 191-201.

[4] R. Redheffer and P. Volkmann, 'Die Funktionalgleichung $f(x)+\max \{f(y), f(-y)\}=\max \{f(x+y)$, $f(x-y)$ \}', in: General Inequalities, 7 (Oberwolfach, 1995), International Series of Numerical Mathematics, 123 (Birkhäuser, Basel, 1997), pp. 311-318.

[5] A. Simon (Chaljub-Simon) and P Volkmann, 'Caractérisation du module d'une fonction à l'aide d'une équation fonctionnelle', Aequationes Math. 47 (1994), 60-68.

BARBARA PRZEBIERACZ, University of Silesia, ul. Bankowa 14, 40-007 Katowice, Poland

e-mail: barbara.przebieracz@us.edu.pl 